\title{
Differential toll-like receptor activation in lung ischemia reperfusion injury
}

\author{
Patrick Phelan, MD, ${ }^{a}$ Heather E. Merry, MD, ${ }^{a}$ Billanna Hwang, MPH, ${ }^{\mathrm{a}, \mathrm{b}}$ and Michael S. Mulligan, MD ${ }^{\mathrm{a}, \mathrm{b}}$
}

\section{ABSTRACT}

Objective: The requirement for toll-like receptors (TLRs) in lung ischemia reperfusion injury (LIRI) has been demonstrated but not fully characterized. Previously, we reported that TLR-4 is required by alveolar macrophages but not pulmonary endothelial or epithelial cells for development of LIRI. Additionally, we demonstrated differential patterns of mitogen-activated protein kinase (MAPK) activation and cytokine release in these cell types during LIRI. Here, we sought to determine whether these differences in activation responses are related to cell-specific TLR activation requirements.

Methods: Primary cultures of alveolar macrophages, pulmonary endothelial, and immortalized epithelial cells were pretreated with TLR-2 or TLR-4 short interference RNA (ribonucleic acid) before hypoxia and reoxygenation. Cell lysates and media were analyzed for receptor knockdown, MAPK activation, and cytokine production. Rats were pretreated with TLR-2 or TLR-4 short interference RNA before lung ischemia reperfusion and changes in lung vascular permeability were assessed.

Results: Knockdown of TLR-2 in alveolar macrophages did not affect MAPK phosphorylation or cytokine secretion. Conversely, TLR-2 knockdown in pulmonary endothelial and epithelial cells demonstrated significant reductions in extracellular signal-regulated kinase $1 / 2$ activation and cytokine secretion. The lung permeability index in LIRI was decreased by TLR-4 but not TLR-2.

Conclusions: Differential TLR signaling and MAPK activation in response to LIRI seem to be cell specific. Short interference RNA provides an outstanding tool for examination of the underlying mechanism. (J Thorac Cardiovasc Surg 2015;149:1653-61)

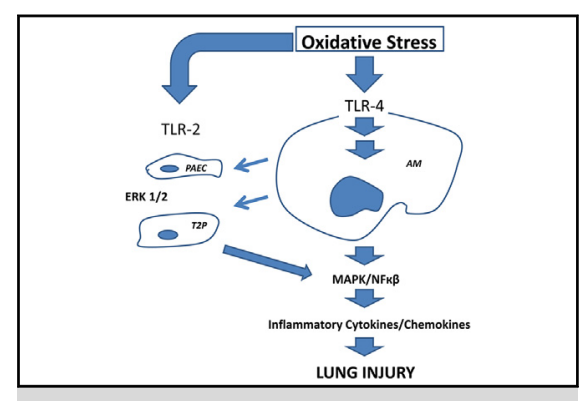

Overview of toll-like receptor activation in lung ischemia reperfusion injury.

\section{Central Message}

Lung ischemia reperfusion injury results from the activation of toll-like receptor-4 in alveolar macrophages and toll-like receptor-2 in endothelial and epithelial cells. This discovery allows for a greater understanding of the interactions between these cell types as well as their differential response to ischemia reperfusion.

\section{Perspective}

The discovery of differential cellular activation in lung ischemia reperfusion injury allows for the exploitation of cellular differences to design precise therapeutics. Ultimately, this work seeks to devise strategies to prevent innate immunity mediated ischemia-reperfusion injury and still allow for the appropriate response to microbial infection.

See Editorial Commentary page 1662.
Despite improvements in organ preservation and donor and recipient management, lung ischemia reperfusion injury (LIRI) remains a significant clinical problem after lung transplantation. ${ }^{1,2} \mathrm{Up}$ to $25 \%$ of lung transplant recipients develop significant reperfusion injury in the hours after

\footnotetext{
From the Department of Surgery, ${ }^{\text {a }}$ University of Washington School of Medicine, Seattle; and Center for Lung Biology, ${ }^{\mathrm{b}}$ University of Washington, Seattle, Wash. This work was supported by NIH RO1 Role of TLR-4 in Lung Reperfusion Injury, and by the National Institutes of Health, (Bethesda, Md) grant R01HL093097.

Received for publication Oct 10, 2014; revisions received Feb 10, 2015; accepted for publication Feb 19, 2015; available ahead of print April 21, 2015.

Address for reprints: Billanna Hwang, MPH, Division of Thoracic Surgery, University of Washington, Center for Lung Biology, 850 Republican St, Box 358052, WA 98109 (E-mail: bhwang@u.washington.edu). 0022-5223/\$0.00

Published by Elsevier Inc. on behalf of The American Association for Thoracic Surgery

http://dx.doi.org/10.1016/j.jtcvs.2015.02.045
}

implantation. ${ }^{3}$ This complication increases the risk of acute rejection, adversely affects early postoperative mortality, and is the strongest acute risk factor for obliterative bronchiolitis. ${ }^{4,5}$ Characterization of the cell-specific inflammatory signaling events in LIRI is a crucial step toward identifying strategies and treatments aimed at ameliorating LIRI and improving outcomes.

Work in vitro has demonstrated that, in response to oxidative stress, activation of the mitogen-activated protein kinases (MAPKs), p38, and c-Jun N-terminal kinase, is discretely associated with the alveolar macrophage. ${ }^{6}$ Additionally, we have demonstrated the importance of early activation of the alveolar macrophage in the development of LIRI. ${ }^{7}$ Conversely, activation of extracellular signalregulated kinase $1 / 2$, but not p38 or c-Jun $\mathrm{N}$-terminal kinase, occurs in pulmonary artery endothelial cells and 


\section{Abbreviations and Acronyms \\ CINC = cytokine-induced neutrophil chemoattractant \\ LIRI = lung ischemia reperfusion injury \\ MAPK $=$ mitogen-activated protein kinase \\ RPMI $=$ Roswell Park Memorial Institute \\ siRNA $=$ short interference ribonucleic acid \\ TLR $=$ toll-like receptor}

type 2 pneumocytes (nonalveolar macrophage). ${ }^{6,7}$ In addition, these discrete, cell-specific patterns of MAPK activation in vitro have been demonstrated in an in situ model of left LIRI using immunohistochemistry. ${ }^{6}$ This differential pattern of MAPK activation in alveolar macrophages versus other cell types suggests alternative requirements for initiation of the proinflammatory signaling cascade in response to oxidative stress. Although MAPK activation is centrally important in LIRI, upstream signaling is likely what renders differentiated cellular responses.

Toll-like receptors (TLRs) are an evolutionarily conserved family of pattern-recognition receptors critical to innate immunity. Their ability to sense alarm and initiate inflammation makes them excellent candidates for early signaling in LIRI. Toll-like receptor-4 is activated by a wide array of signals, including stressed, necrotic, or injured tissue, but its response to lipopolysaccharide has been the most thoroughly characterized. ${ }^{8-10}$ Activation of TLR-4 in response to lipopolysaccharide stimulation initiates downstream recruitment and activation of specific adaptor proteins, signaling kinases, and transcription factors, ultimately resulting in the transcription and secretion of proinflammatory cytokines and chemokines. ${ }^{11,12}$ In addition, TLR-4 has been implicated as a key modulator in several models of ischemia and reperfusion. Deletion or pharmacologic antagonism of TLR-4 has been shown to reduce injury severity in cardiac, hepatic, renal, and cerebral models of ischemia reperfusion. ${ }^{13-16}$

Shimamoto and colleagues and Merry and colleagues $^{17,18}$ recently demonstrated that LIRI requires TLR-4, using knockout mice, a finding that supports our work using short interference ribonucleic acid (siRNA) for target molecular deletion of TLR-4. We demonstrated that TLR-4 knockdown in the alveolar macrophage protects against LIRI through decreased MAPK phosphorylation, proinflammatory cytokine production, and a corresponding reduction in the permeability index. However, cytokine production remained elevated in pulmonary artery endothelial cells and type 2 pneumocytes in response to oxidative stress, despite TLR-4 knockdown. ${ }^{18}$ This lack of response to TLR-4 knockdown in these cell types, along with the differential MAPK phosphorylation in these cell types, suggests a different initial site or sequence of inflammatory activation.

A similar array of ligands is exhibited by TLR-2, including both bacterial products, such as lipotheichoic acid, and host molecules. Furthermore, TLR-2 is known to have an important role in the initiation of inflammatory responses and the development of ischemic injury in the heart and kidney. ${ }^{19,20}$ In the kidney, TLR-2 is strongly expressed within glomerular endothelial cells and epithelial cells of Bowman's capsule. Mice with TLR-2 knockout demonstrate an attenuated injury response to renal ischemia and reperfusion. ${ }^{19}$ No reports to date have examined the role of TLR-2 in the mediation of LIRI, but TLR-2 is expressed on bronchial epithelial cells, and has been shown to be involved in various organ-specific models of ischemia reperfusion. ${ }^{13-16,21,22}$ Therefore, TLR-2 is a candidate for the initial site of inflammatory signaling activation in pulmonary artery endothelial cells and type 2 pneumocytes in response to oxidative stress.

The purpose of this study was to determine whether the differential responses to oxidative stress among alveolar macrophages, pulmonary artery endothelial cells, and type 2 pneumocytes relate to cell-specific TLR activation patterns leading to differential downstream signaling responses. Previous in vivo studies have demonstrated that intravenous administration of siRNA transfects alveolar macrophages, but not pulmonary artery endothelial cells or type 2 pneumocytes, necessitating the use of in vitro studies to investigate the effects of TLR-2 deletion on these nonalveolar macrophage cell types in response to oxidative stress (unpublished data). Using siRNA to achieve targeted molecular deletion of TLR-2 and TLR-4 in cultured alveolar macrophages, pulmonary artery endothelial cells, and type 2 pneumocytes, we examined the cell-specific requirements for TLR activation in LIRI. Additionally, a well-developed model of LIRI in the rat was utilized for further characterization.

\section{METHODS}

\section{Short Interference Ribonucleic Acid}

The siRNAs used in this study were designed (Invitrogen Corporation, Carlsbad, Calif) and implemented as previously described. ${ }^{18}$ A single siRNA duplex targeting TLR-4 was used, based on prior work demonstrating its efficacy of uptake and TLR-4 knockdown, in the cell types of interest and the animal model. ${ }^{18}$ Three unique, nonoverlapping siRNA duplexes were designed to target TLR-2. A noncoding, scrambled siRNA sequence was used as a control. For our in vitro experiments, 100 pmol of oligonucleotide was diluted in $50 \mu \mathrm{l}$ of growth media, per well, in a 12-well culture plate, incubated for 15 minutes, and combined with either $3 \mu \mathrm{l}$ of lipofectamine (Lipofectamine 2000, Invitrogen Corporation, Carlsbad, Calif) diluted in $50 \mu \mathrm{l}$ of growth media (for alveolar macrophages), or $1 \mu \mathrm{l}$ of lipofectamine diluted in $50 \mu \mathrm{l}$ of growth media (for type 2 pneumocytes and pulmonary artery endothelial cells). This siRNA-lipid mixture replaced the cell culture media, and cells were incubated with this mixture for a minimum of 6 hours. 


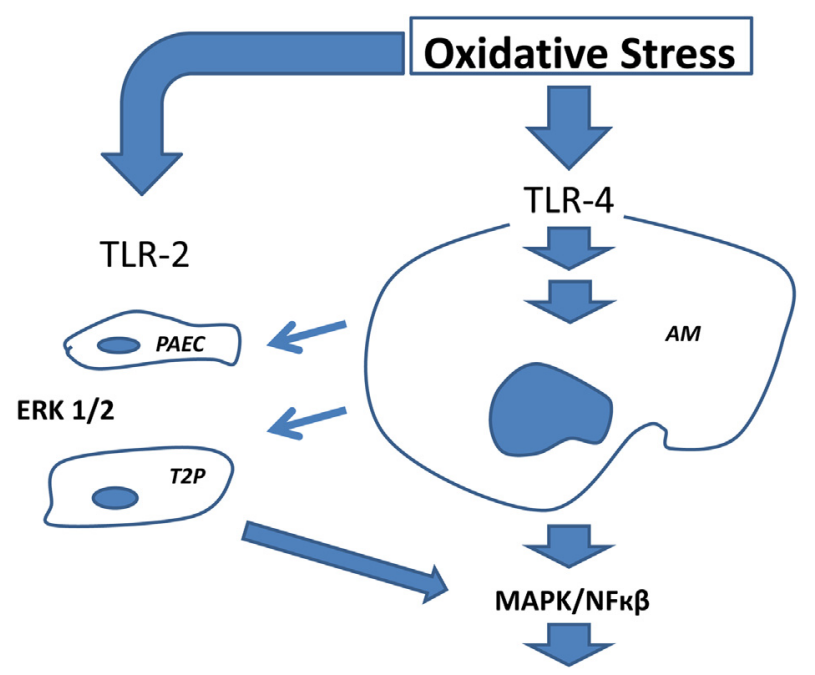

Inflammatory Cytokines/Chemokines

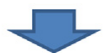

\section{LUNG INJURY}

FIGURE 1. Overview of TLR activation in lung ischemia reperfusion injury; oxidative stress is initially transduced through TLR-4 activation in $\mathrm{AM}$, triggering an inflammatory signaling cascade, leading to the development of lung injury. Products of AM activation augment and amplify the inflammatory responses, via TLR-2 pathways from both PAEC and T2P. TLR, Toll-like receptor; $A M$, alveolar macrophages; $P A E C$, pulmonary artery endothelial cells; $E R K$, extracellular signalregulated kinase; $T 2 P$, type 2 pneumocytes; $M A P K$, mitogen-activated protein kinase; $N F \mathrm{~K} \beta$, nuclear factor kappa beta.

For our in vivo experiment, $10 \mathrm{nM}$ of siRNA was diluted in $250 \mu \mathrm{l}$ of sterile saline and combined with $100 \mu \mathrm{l} / \mathrm{kg}$ of lipid vector diluted in $250 \mu \mathrm{l}$ of sterile saline and administered twice, at 24 hours and 48 hours before undergoing our ischemia reperfusion protocol. The siRNA-lipid complex was incubated for 15 minutes and rapidly injected into the penile vein. For TLR-2 knockdown, TLR-2 sequence-1 was used, because it yielded the greatest knockdown efficacy. The antisense sequences used for the siRNA were as follows:

- TLR-4 sequence 2: 5'-UCU GCU CCA CCA UUG GGC UUA ACC A-3' - TLR-2 sequence 1: 5'-GAC AAC AUC AUU GAU UCC AUC GAA A-3' - TLR-2 sequence 2: $5^{\prime}$-GGA GCU AGG UAA AGU AGA AAC GGU A-3' - TLR-2 sequence 3: 5'-CCU CGG CUC CAA GAG CUG UAU AUU U-3'

- Control: noncoding siRNA, medium guanine-cytosine content.

\section{Ischemia Reperfusion Protocol}

Pathogen-free adult male Long-Evans rats (Harlan Sprague Dawley, Inc, Indianapolis, Ind), weighing 275 to $300 \mathrm{~g}$, were used for all in vivo experiments. All procedures were performed in accordance with the guidelines of the Institutional Animal Care and Use Committee at the University of Washington.

A well established, in situ warm ischemia reperfusion model was utilized as previously described. ${ }^{18,23}$ Briefly, rodents were anesthetized with $30 \mathrm{mg}$ of intraperitoneal pentobarbital, and a 14-gauge angiocatheter was placed under direct vision into the trachea through a midline neck incision. The catheter was secured and the animal was placed on a Harvard Rodent Ventilator (Harvard Apparatus, Inc, Holliston, Mass). Ventilator settings were standardized with an inspired oxygen content of $60 \%, 2$ $\mathrm{cm}$ water of positive end-expiratory pressure, and a respiratory rate of 80. A left thoracotomy was performed, and the left lung was mobilized.
Fifty units of heparin $(500 \mu \mathrm{L})$ was administered via the penile vein and allowed to circulate for 5 minutes. A noncrushing clamp was placed across the left lung hilum, occluding the pulmonary artery, vein, and mainstem bronchus.

After 90 minutes, the clamp was removed, and the left lung was allowed to reventilate and reperfuse for 4 hours, during which $500 \mu \mathrm{L}$ of warm saline was injected subcutaneously every hour to maintain hydration. At the end of reperfusion, a midline laparotomy and sternotomy were performed. Blood samples were taken from the inferior vena cava, and the animals were killed. The heart-lung block was excised, and the pulmonary circulation was flushed with $20 \mathrm{~mL}$ of saline. After this step, the left lung was separated from other mediastinal structures.

\section{In Vivo Experimental Groups}

Four experimental groups were studied: negative control animals (noncoding siRNA and no ischemia reperfusion protocol) and positive control animals (noncoding siRNA and ischemia reperfusion protocol). The 2 remaining experimental groups received either TLR-2 or TLR-4 siRNA before undergoing the ischemia reperfusion protocol.

\section{Alveolar Macrophage Harvest}

Alveolar macrophages were isolated and cultured as described previously. ${ }^{7,24}$ Briefly, the heart-lung block was subsequently excised via a median sternotomy. Intratracheal lavage was performed with $50 \mathrm{~mL}$ of cold phosphate-buffered saline as previously described. The effluent was centrifuged at $1500 \mathrm{~g}$ for 10 minutes, and the cell pellet was resuspended in serum-free RPMI medium (Roswell Park Memorial Institute; Gibco BRL custom primers, Life Technologies, Rockville, Md). Cell counts and viability were assessed by standard trypan blue exclusion methods. Cells were then plated at a density of 1,000,000 cells per well.

\section{Type 2 Pneumocytes}

A rat type 2 pneumocyte cell line, RLE-6TN (American Tissue Cell Company, Manassas, Va) was maintained in Hams F-12 (Life Technology, Rockville, Md) culture media containing 10\% heat-inactivated fetal bovine serum. The type 2 pneumocytes were cultured in 12-well plates at a density of 200,000 per $\mathrm{mL}$. Cell counts and viability were assessed by a standard trypan blue exclusion technique, and media were replenished every 48 hours until confluence was achieved.

\section{Rat Pulmonary Artery Endothelial Cell Culture}

The heart-lung block was harvested, and bronchoalveolar lavage was performed as described earlier, except 21-day-old rats were used. Pulmonary artery endothelial cells were isolated, cultured, and purified as described elsewhere by our group. ${ }^{25,26}$ Endotracheal lavage was performed in a similar fashion as described earlier for the alveolar macrophage harvest, except phosphate-buffered saline containing 0.25 $\mathrm{mM}$ ethylenediaminetetraacetic acid was used for the lavage fluid to deplete alveolar macrophages. Peripheral lung was removed in 2-mm strips from all lung lobes. Tissue was minced, rinsed in RPMI media, transferred to a dispase $(10 \mathrm{mg} / \mathrm{mL})$ solution, and incubated for 60 minutes at $37^{\circ} \mathrm{C}$. The cell suspension was homogenized and incubated for 5 minutes at $37^{\circ} \mathrm{C}$. Complete media $(10 \mathrm{ml})$ containing $10 \%$ fetal bovine serum were added, to terminate the reaction, and the cellular suspension was filtered through a $100-\mu \mathrm{m}$ mesh. The filtrate was centrifuged at $800 \mathrm{~g}$ for 8 minutes, resuspended in supplemented RPMI media, and plated on gelatin-coated culture dishes. The media were changed every 48 hours during incubation until they were confluent. Cells were labeled for 8 hours with $4 \mu \mathrm{g} / \mathrm{mL}$ of acetylated low-density lipoproteins, which bind selectively to endothelial cells. Cells were separated using flow cytometry (FAC Star Plus flow cytometer, Becton, Dickinson, and Co, San Jose, Calif) to maintain a pure culture of endothelial cells. All cells used were from passages 4 to 8 , to obtain optimal results. Cells were plated in 12-well plates at a concentration of 500,000 cells $/ \mathrm{mL}$, and used for experiments when confluent. 


\section{Hypoxia and Reoxygenation Protocol}

Hypoxia and reoxygenation was performed as described elsewhere. $^{24,26}$ Briefly, once type 2 pneumocytes or pulmonary artery endothelial cells reached confluence, or alveolar macrophages had quiesced for $\geq 6$ hours, the cells were placed in a hypoxic chamber (fraction of inspired oxygen: $0.5 \%$ ) for 90 minutes, and afterward, placed in a normoxic (fraction of inspired oxygen: $21 \%$ ) incubator for up to 4 hours. Negative controls remained in the normoxic incubator for up to 6 hours. After 15 minutes of reoxygenation, total protein was harvested for $\mathrm{p} 38$, c-Jun N-terminal kinase activation; after 1 hour of reoxygenation, total protein was harvested for extracellular signalregulated kinase 1/2 activation. After 4 hours of reoxygenation, media were harvested and analyzed for cytokine-induced neutrophil chemoattractant (CINC) secretion by enzyme-linked immunosorbent assay.

\section{In Vitro Experimental Groups}

To determine sequence-specific knockdown efficacy, alveolar macrophages were pretreated with either TLR-2 sequence-1, sequence-2, or sequence-3 siRNA, and were compared with positive controls that received pretreatment with noncoding siRNA before undergoing hypoxia and reoxygenation. Negative control cells (noncoding siRNA and no protocol) and positive control (noncoding siRNA and hypoxia and reoxygenation protocol) were used. The 2 remaining experimental groups received either TLR- 2 or TLR-4 siRNA before undergoing the hypoxia and reoxygenation protocol.

\section{Western Blot Analysis}

Total protein was extracted from whole left lung homogenates and cultured cells. Protein $(40 \mu \mathrm{g})$ was electrophoresed on $10 \%$ sodium dodecylsulfate-polyacrylamide gel and transferred to a polyvinylidene difluoride membrane (Amersham Hybond PVDF blotting membrane; Amersham Pharmacia Biotech Inc, Piscataway, NJ). The membrane was blocked for 1 hour at room temperature with $5 \%$ blotto and then incubated with either a phosphospecific extracellular signal-regulated kinase $1 / 2$ (threonine 202 and tyrosine 204) antibody, a phosphospecific p38 (threonine 180 and tyrosine 182) antibody, a phosphospecific c-Jun N-terminal kinase (threonine 183/ tyrosine 185) polyclonal antibody, or a TLR-2-specific antibody (TLR2 antibody \#2229, Cell Signaling Technology, Inc, Beverly, Mass) overnight at $4^{\circ} \mathrm{C}$. After this step, the membrane was incubated with a horseradish-peroxidase conjugated secondary antibody, for 1 hour at room temperature. The blot was developed using the SuperSignal West Pico Chemiluminescent Substrate (Pierce Chemical Company, Rockford, Ill), and exposed on Kodak film. Densitometry was performed using Image $\mathbf{J}$ (version 1.2; Media Cybernetics, Inc, Rockville, Md).

\section{Protein Analysis for Cytokine Content by Enzyme- Linked Immunosorbent Assay}

After 4 hours of in vitro reoxygenation, media were collected and processed as described elsewhere. ${ }^{23,24}$ Media were analyzed for CINC content via sandwich enzyme-linked immunosorbent assay, as described elsewhere. ${ }^{23,24}$

\section{Lung Permeability Index}

To quantitate lung injury, a permeability index of the lung was determined, as described elsewhere. ${ }^{23}$ In the next step, ${ }^{125}$-radiolabeled bovine serum albumin (NEN Life Science Products, Inc, Boston, Mass), titrated to an activity of 800,000 counts per minute per dose, was intravenously injected in a final volume of $500 \mu 1$ of $1 \%$ bovine serum albumin -phosphate-buffered saline solution 5 minutes before removal of the hilar clamp. After 4 hours of reperfusion, the radioactivity counts were quantitated in the left lung, and in $1 \mathrm{~mL}$ of inferior vena cava blood, using a scintillation counter. They were calculated as: permeability index $=$ left lung $/ 1 \mathrm{~mL}$ blood (both in counts per minute). This ratio corrects for any variation in systemic blood levels of radioactivity and provides a reproducible measure of lung microvascular permeability.

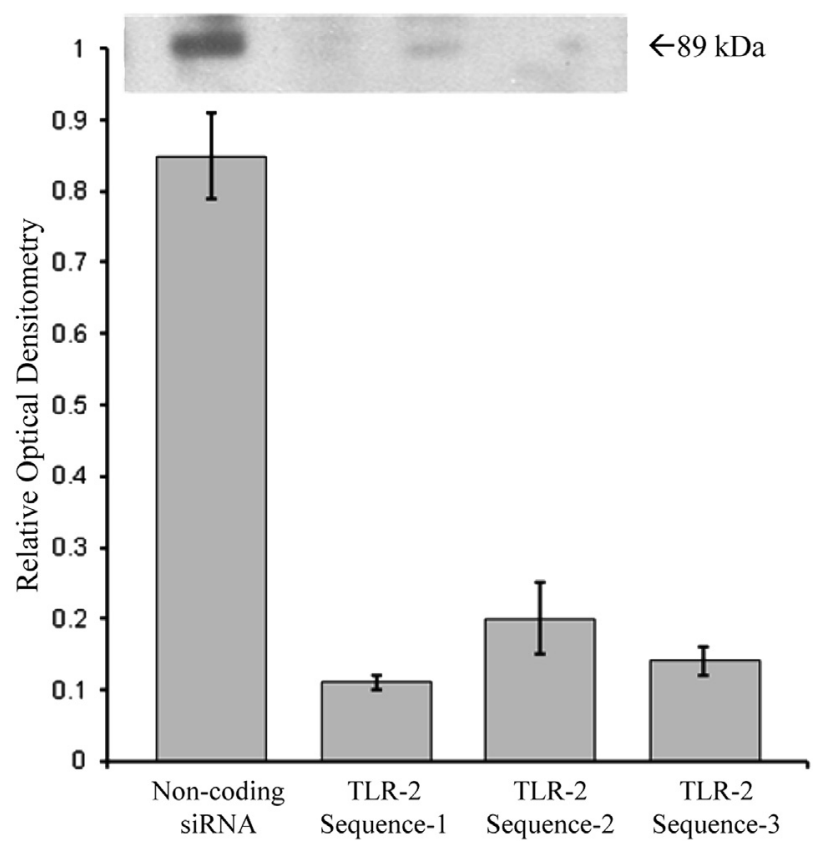

FIGURE 2. Sequence-specific TLR-2 knockdown in vitro; primary cultures of alveolar macrophages pretreated with TLR-2 siRNA sequence-1, sequence- 2 , and sequence- 3 demonstrated a reduction in TLR-2 expression by $87 \%(P<.001), 78 \%(P<.01)$, and $84 \%$ $(P<.001)$, respectively, compared with alveolar macrophages pretreated with noncoding siRNA, as assessed by relative optical densitometry of Western blot analysis ( $\mathrm{n}=12$ wells per group). siRNA, Short interference ribonucleic acid; $T L R$, toll-like receptor.

\section{Statistical Analysis}

All data are presented as mean values \pm the standard error of the mean. Comparisons among groups were made using 1-way analysis of variance. Bonferroni's method was used to adjust for multiple comparisons. A post-hoc 2-tailed Student $t$ test was performed to assess statistical differences between individual groups.

\section{RESULTS}

\section{Sequence-Specific Toll-Like Receptor-2 Knockdown In Vitro}

Primary cultures of alveolar macrophages pretreated with TLR-2 siRNA sequence-1, sequence-2, and sequence- 3 demonstrated a reduction in TLR-2 expression by $87 \%$ $(P<.001), 78 \%(P<.01)$, and $84 \%(P<.001)$, respectively, compared with alveolar macrophages pretreated with noncoding siRNA, as assessed by relative optical densitometry of Western blot analysis (Figure 1). For all subsequent experiments, TLR-2 sequence- 1 was used, as it was the most efficacious at achieving TLR-2 knockdown.

\section{Efficacy of Toll-Like Receptor-2 Knockdown In Vitro}

Knockdown of TLR-2 in siRNA pretreated alveolar macrophages, pulmonary artery endothelial cells, and type 2 pneumocytes was confirmed by Western blot analysis with densitometry. Preteatment with TLR-2 siRNA 

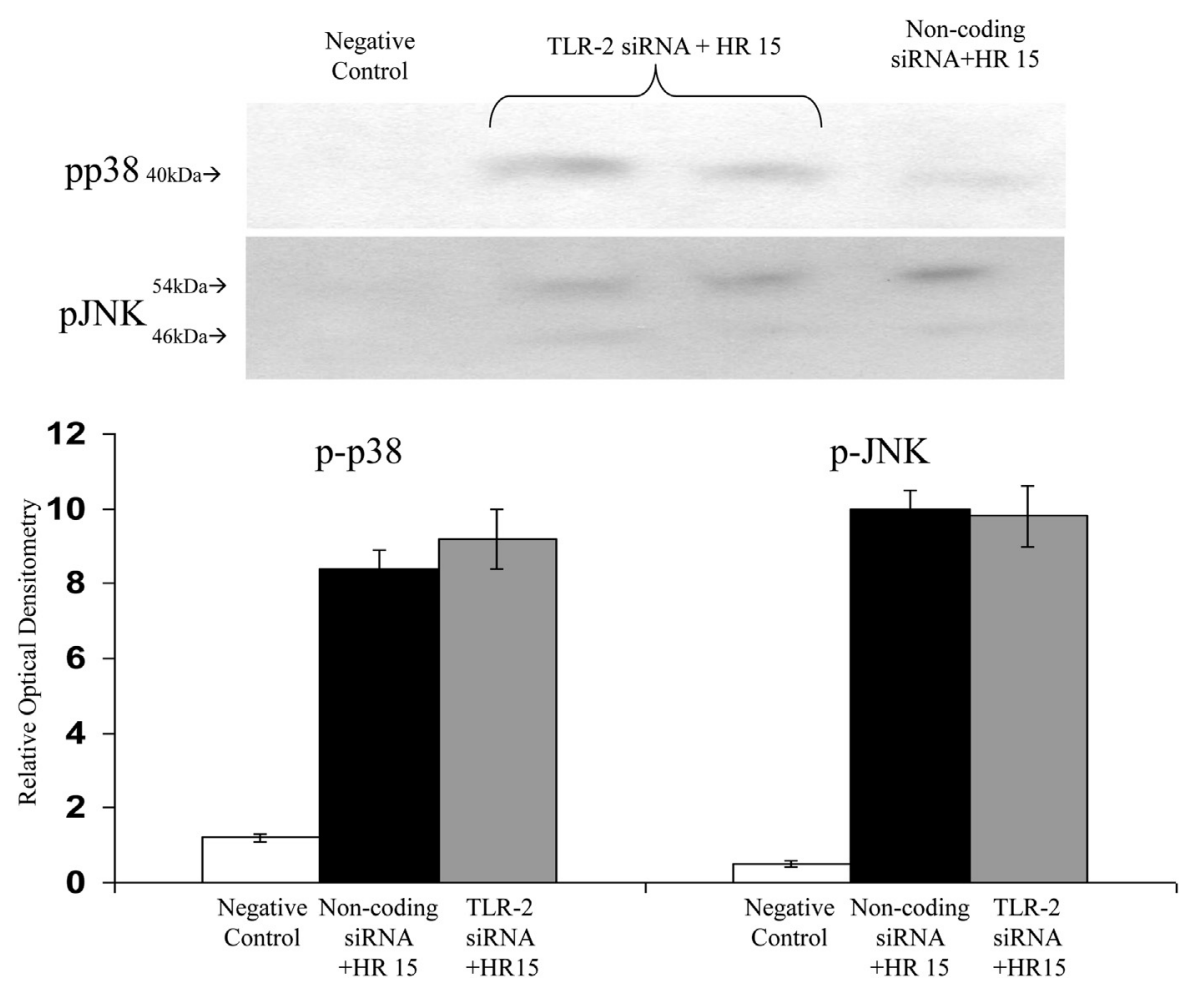

FIGURE 3. Mitogen-activated protein kinase phosphorylation after TLR-2 knockdown in alveolar macrophages; phosphorylation of p38 and JNK (pJNK) persisted $(P>.02$ and $P>.02$, respectively) in alveolar macrophages pretreated with TLR-2 siRNA, compared with noncoding siRNA-pretreated control alveolar macrophages after undergoing HR15, as assessed by relative optical densitometry of Western blot analysis ( $\mathrm{n}=12$ wells per group). TLR, Toll-like receptor; siRNA, short interference ribonucleic acid; $H R$, hypoxia and reoxygenation; $p p 38$, phosphorylation of p38; $J N K$, c-Jun N-terminal protein kinases; pJNK, phosphorylation of JNK.

sequence- 1 resulted in a reduction in TLR-2 expression by $86 \%(P<.01)$ in alveolar macrophages, $77 \%(P<.01)$ in pulmonary artery endothelial cells, and $82 \%(P<.01)$ in type 2 pneumocytes, compared with noncoding siRNA pretreated cells, as assessed by relative optical densitometry of Western blot analysis (Figure 2).

\section{Phosphorylation After Toll-Like Receptor-2 Knockdown In Vitro}

Mitogen-activated protein kinase phosphorylation persisted ( $P>.02$ and $P>.02$, respectively) for phosphorylated p38 and c-Jun N-terminal kinase in alveolar macrophages pretreated with TLR-2 siRNA, compared with noncoding siRNA-pretreated control alveolar macrophages (Figure 3). Conversely, extracellular signal-regulated kinase phosphorylation was significantly diminished in TLR-2 siRNA-pretreated pulmonary artery endothelial cells $(P<.01)$ and type 2 pneumocytes $(P<.01)$, compared with noncoding siRNA-pretreated control cells (Figure 4). Additionally, no alteration occurred $(P>.02)$ in extracellular signal-regulated kinase phosphorylation in pulmonary artery endothelial cells pretreated with TLR-4 siRNA, compared with noncoding siRNA-pretreated control pulmonary artery endothelial cells (Figure 5).

\section{Cytokine Production After Toll-Like Receptor-2 and Toll-Like Receptor-4 Knockdown In Vitro}

Toll-like receptor-2 knockdown did not alter $(P>.02)$ secretion of the inflammatory cytokine CINC in alveolar macrophages, compared with noncoding siRNA-pretreated control alveolar macrophages (Figure 6). Conversely, TLR-2 knockdown decreased CINC secretion in pulmonary artery endothelial cells and type 2 pneumocytes by $76 \%$ $(P<.001)$ and 78\% $(P<.001)$, respectively, compared with noncoding siRNA-pretreated control cells. Cultured alveolar macrophages pretreated with TLR-4 siRNA showed a significant reduction in CINC secretion $(P<.0001)$, compared with noncoding siRNA-pretreated control alveolar macrophages. However, TLR-4 knockdown did not affect CINC secretion in pulmonary artery endothelial cells or type 2 pneumocytes $(P>.02$ and $P>.02$, respectively), compared with noncoding siRNApretreated control cells.

\section{Permeability Index After Toll-Like Receptor-2 and Toll-Like Receptor-4 Knockdown In Vivo}

With TLR-4 siRNA pretreatment, lung vascular permeability was reduced by $85 \%(P<.001)$, compared with noncoding siRNA-pretreated control animals after 

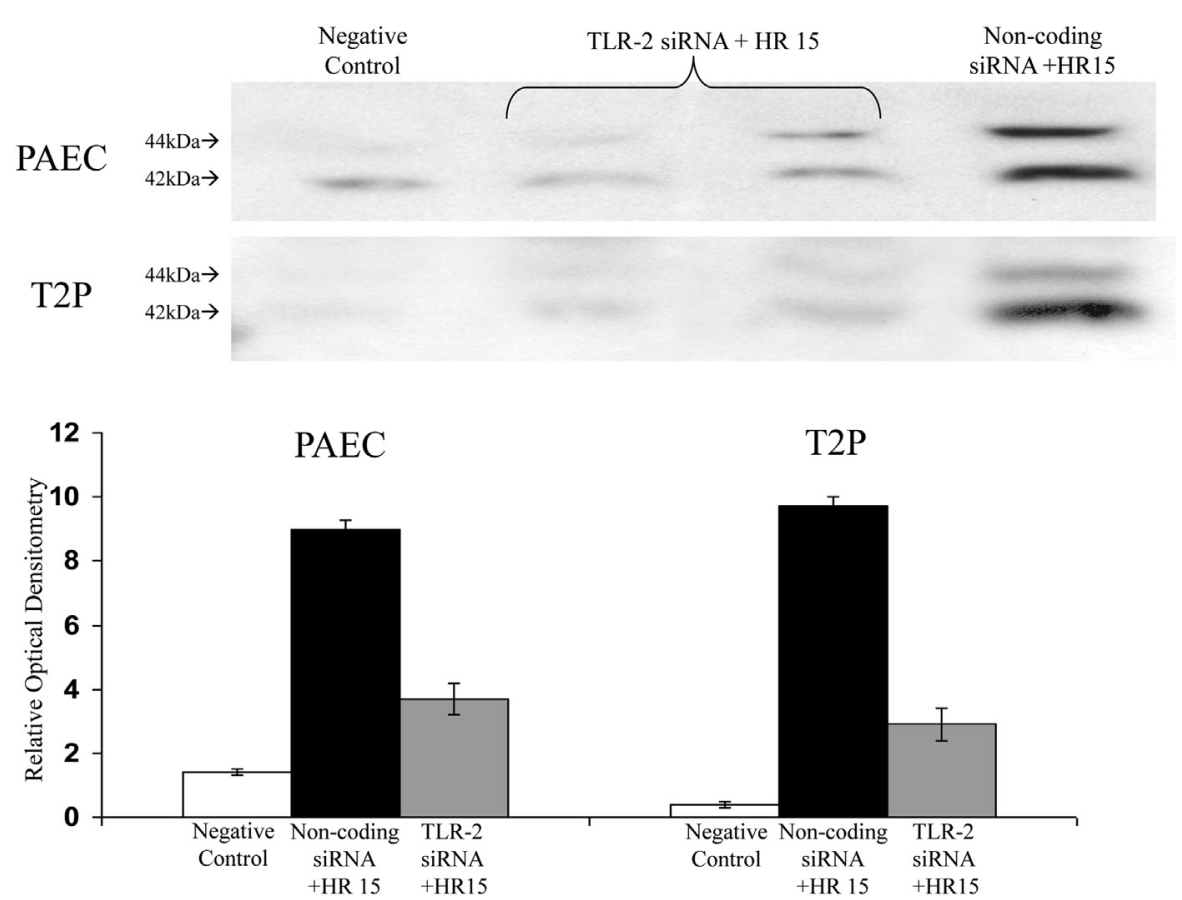

FIGURE 4. Extracellular signal-regulated kinase phosphorylation after TLR-2 knockdown in vitro; pulmonary artery endothelial cells (PAEC) and T2P pretreated with TLR-2 siRNA demonstrated significant reduction in ERK phosphorylation $(P<.01$ and $P<.01$, respectively), compared with noncoding siRNA-pretreated control cells after undergoing HR15, as assessed by relative optical densitometry of Western blot analysis ( $\mathrm{n}=12$ wells per group). TLR, Toll-like receptor; siRNA, short interference ribonucleic acid; $H R$, hypoxia and reoxygenation; PAEC, pulmonary artery endothelial cells; $T 2 P$, type 2 pneumocytes.

90 minutes of ischemia followed by 4 hours of reperfusion. In fact, no statistical difference $(P>.02)$ was found in the vascular permeability in animals pretreated with TLR-4 siRNA compared with those used as negative controls. In contrast, pretreatment with TLR-2 siRNA did not confer protection $(P>.02)$ from subsequent ischemia reperfusion injury, compared with noncoding siRNA-pretreated animals after 90 minutes of ischemia followed by 4 hours of reperfusion (Figure 7).

\section{DISCUSSION}

These experiments establish differential roles for TLR-2- and TLR-4-dependent proinflammatory signaling in response to oxidative stress in the lung. Although we have previously shown that TLR-4 activation in the alveolar macrophage is centrally important, these data suggest a novel role for TLR-2 in the proinflammatory response of pulmonary artery endothelial cells and type 2 pneumocytes to oxidative stress in the lung. Knockdown of TLR-4, but not TLR-2, in the alveolar macrophage in vivo led to marked protection from lung injury. The protection seen with TLR-4 knockdown in vivo was likely secondary diminished TLR-4-mediated MAPK phosphorylation in alveolar macrophages, as our prior work has shown. ${ }^{18}$

The lack of protective response to TLR-2 siRNA administration in vivo may be due to several factors. Prior in vivo studies have shown that intravenous administration of TLR-4 siRNA resulted in preferential uptake by alveolar macrophages, not pulmonary artery endothelial cells or type 2 pneumocytes. Therefore, when TLR-2 siRNA is administered intravenously, it may not be taken up by pulmonary artery endothelial cells or type 2 pneumocytes, but instead be taken up by the alveolar macrophages. Alternatively, although TLR-2 knockdown diminished extracellular signal-regulated kinase phosphorylation and resultant downstream proinflammatory signaling in response to oxidative stress in cultured type 2 cells and pulmonary artery endothelial cells, TLR-4-dependent proinflammatory signaling in the alveolar macrophage may be the predominant signaling response to oxidative stress. Given this possibility, protection from LIRI may not be achieved without TLR-4 knockdown in alveolar macrophages.

Toll-like receptor-4 knockdown in the pulmonary artery endothelial cells and type 2 pneumocytes did not diminish inflammatory cytokine production or extracellular signal-regulated kinase $1 / 2$ phosphorylation. These results support the hypothesis that TLR-2 regulates the response to oxidative stress in these cell types, whereas TLR-4 does so in the alveolar macrophage. Further studies evaluating the effects of simultaneous TLR-2 and TLR-4 knockdown in vivo may be warranted to confirm that simultaneous knockdown and inhibition of both proinflammatory 

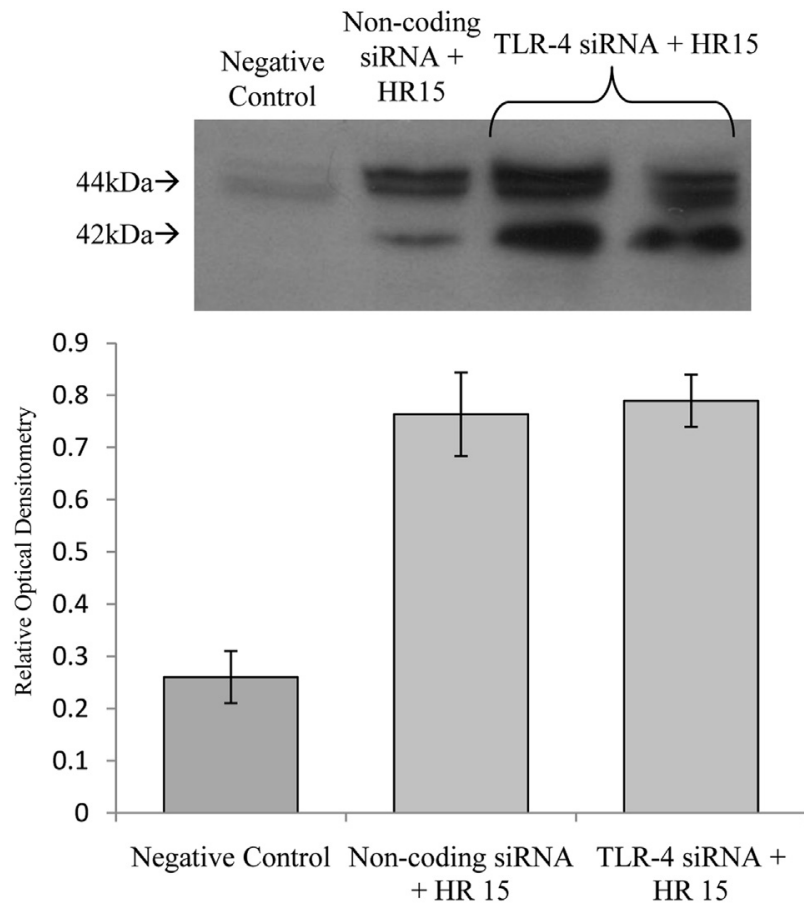

FIGURE 5. Extracellular signal-regulated kinase phosphorylation after TLR-2 knockdown of PAEC; no alteration occurred $(P>.02)$ in extracellular signal-regulated kinase phosphorylation in PAEC pretreated with TLR-4 siRNA, compared with noncoding siRNA-pretreated control PAEC after undergoing HR15, as assessed by relative optical densitometry of Western blot analysis ( $\mathrm{n}=12$ wells per group). siRNA, Short interference ribonucleic acid; $H R$, hypoxia and reoxygenation; $T L R$, toll-like receptor; $P A E C$, pulmonary artery endothelial cells.

signaling pathways in these relevant lung types provides greater protection form LIRI. Such studies are limited, however, by the selective inability to transfect type 2 cells and pulmonary artery endothelial cells in vivo.

Investigating ischemia reperfusion injury requires not only pertinent, appropriate animal models, but also cell culture models, to discretely map critical cell-signaling events. Although in vitro studies fall short of reconstituting complex in vivo interactions, they are an important complement to animal studies, as a means to characterize critical signaling events. Our warm hilar occlusion model is specific and reliable for the study of mechanisms of injury related to circumstances in which warm atelectatic ischemia and reperfusion are inevitable in clinical lung transplantation. Our model not only is relevant, but also addresses an important clinical problem that has a very predictable time of onset and allows for practical pretreatment and intervention, unlike most types of acute inflammatory lung injury (ie, acute respiratory distress syndrome).

To address the upstream, cell surface receptor-initiated signaling events in LIRI in vivo and in vitro, we developed a method of specific molecular knockdown, using siRNA targeting TLR-2 and TLR-4. Although in vitro transfection of multiple cell types using siRNA has been widely used, in vivo intravenous delivery and uptake in the lung in a model of LIRI has not yet been reported. ${ }^{27,28}$ This novel approach, although not without limitations, provides a reliable, reproducible, and specific means for targeting candidate molecules involved in LIRI. Furthermore, our method of TLR knockdown using siRNA is consistent between the in vivo and in vitro models, eliminating a methodologic difference between cell culture and animal models. Finally, widely utilized pharmacologic inhibitors have drawbacks, with regard to loss of specificity at higher doses, incomplete blockade, and availability. Such limitations are not relevant concerns with our approach.

Concerns regarding the use of siRNA relate broadly to sometimes-overlapping off-target effects, experimental controls, and specificity. To address these concerns, specificity and methodologic controls must be employed. In our study, several approaches were applied, as follows. Preliminary dose-response experiments provided the minimal effective dose for TLR knockdown $(20 \mathrm{nM}$ in vivo), thereby minimizing dose-related off-target effects. Toxicity control experiments utilized lipofectamine 2000 vector alone in all experiments, which did not induce injury or an inflammatory response alone; negative controls received noncoding siRNA. Three nonoverlapping, independent siRNA sequences were tested for TLR-2 and TLR-4, all of which demonstrated effective target-protein knockdown and the desired phenotype, increasing our confidence that the observed phenotype can be attributed to target-gene knockdown. Additionally, Stealth siRNA (Invitrogen Corporation, Carlsbad, Calif) are designed to ensure that only the antisense strand of the siRNA duplex is processed by the endogenous RNA interference machinery, limiting the potential off-target effects from the sense strand. Finally, we have demonstrated effective target-protein knockdown in the context of the controls previously mentioned.

Although we have previously demonstrated that MAPK activation and inflammatory signaling in the alveolar macrophage in response to oxidative stress is TLR-4 dependent, a similar requirement for TLR-2 activation in the alveolar macrophage does not seem to exist. Moreover, TLR-2 seems to regulate pulmonary artery endothelial cell and type 2 pneumocyte responses to hypoxia and reoxygenation, whereas a similar role for TLR-4 was not demonstrated. Thus, we have identified specific cellular sites of TLR-2 and TLR-4 activation and the downstream effects on signaling. Our novel in vivo and in vitro use of siRNA targeting the lung or resident pulmonary cells provides us with an effective tool for studying the mechanisms, potential ligands, and relevant adaptor proteins responsible for TLR signaling.

These studies are a fundamental step toward identifying potential treatments aimed at improving clinical outcomes 


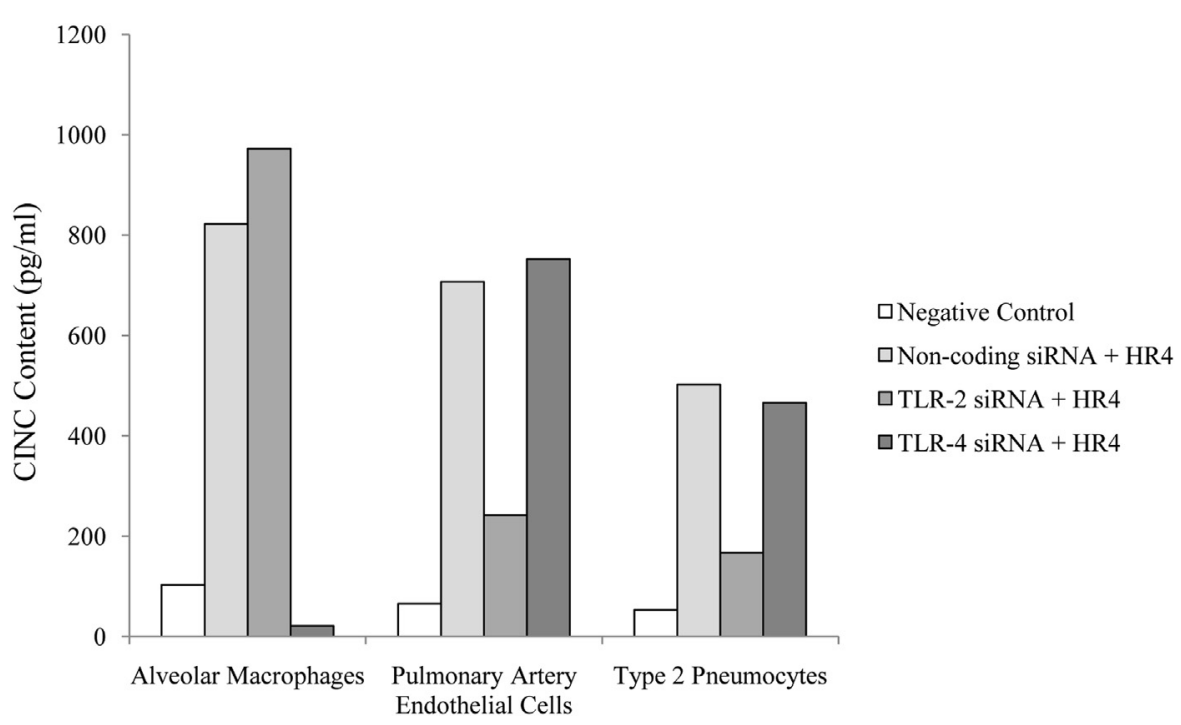

FIGURE 6. Cytokine production after TLR-2 and TLR-4 knockdown in vitro; CINC production is not affected $(P>.02$ and $P>.02$, respectively) by TLR-4 knockdown in pulmonary artery endothelial cells or type 2 pneumocytes, but is reduced by $76 \%(P<.001)$ and $78 \%(P<.001)$, respectively, with TLR-2 knockdown in these cell types, compared with noncoding siRNA-pretreated control cells after undergoing HR4. Conversely, CINC production is not affected $(P>.02)$ by TLR-2 knockdown in alveolar macrophages, but is reduced by $97 \%(P<.001)$ with TLR-4 knockdown in alveolar macrophages, compared with noncoding siRNA-pretreated control cells after undergoing HR4. CINC, Cytokine-induced neutrophil chemoattractant; siRNA, Short interference ribonucleic acid; $H R$, hypoxia and reoxygenation; $T L R$, toll-like receptor.

after lung transplantation. Therapies aimed at blocking a single inflammatory cytokine or signaling molecule such as the MAPKs have failed in both treatments and prophylaxis of LIRI. The current study provides evidence

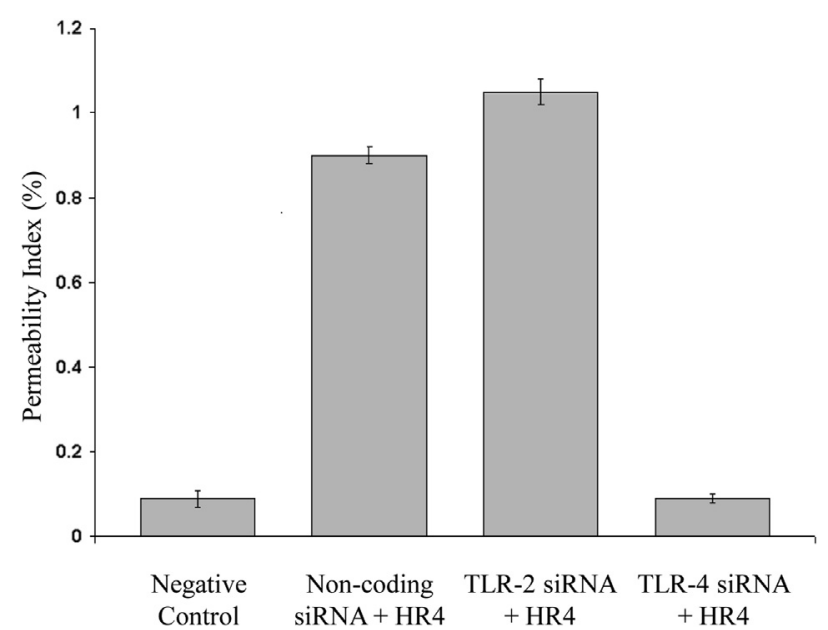

FIGURE 7. Permeability index after TLR-2 and TLR-4 knockdown in vivo; with TLR-4 siRNA pretreatment, the vascular permeability was reduced by $85 \%(P<.001)$, compared with noncoding siRNA-pretreated control animals after undergoing ischemia and reperfusion. No statistical difference was found $(P>.02)$ in the vascular permeability in animals pretreated with TLR-4 siRNA before undergoing ischemia and reperfusion versus negative control animals that did not receive pretreatment or undergo ischemia reperfusion. In contrast, pretreatment with TLR-2 siRNA did not confer protection $(P>.02)$ from subsequent ischemia reperfusion injury, compared with noncoding siRNA-pretreated animals after undergoing ischemia and reperfusion ( $\mathrm{n}=4$ animals per group). siRNA, Short interference ribonucleic acid; $H R$, hypoxia and reoxygenation; $T L R$, toll-like receptor. that TLR signaling pathways play a significant role in the development of LIRI, and interventions at the early stages of these pathways provide potentially attractive novel targets for therapeutic strategies. Additional studies are required to assess the efficacy of TLR-targeted therapies in LIRI before moving to clinical trials.

\section{Conflict of Interest Statement}

Authors have nothing to disclose with regard to commercial support. The content is solely the responsibility of the authors and does not necessarily represent the official views of the National Institutes of Health or its subsidiary institutes and centers.

\section{References}

1. Angel LF, Levine DJ, Restrepo MI, Johnson S, Sako E, Carpenter A, et al. Impact of a lung transplantation donor-management protocol on lung donation and recipient outcomes. Am J Respir Crit Care Med. 2006;174:710-6.

2. Pierre AF, Keshavjee S. Lung transplantation: donor and recipient critical care aspects. Curr Opin Crit Care. 2005;11:339-44.

3. King RC, Binns OA, Rodriguez F, Kanithanon RC, Daniel TM, Spotnitz WD, et al. Reperfusion injury significantly impacts clinical outcome after pulmonary transplantation. Ann Thorac Surg. 2000;69:1681-5.

4. Fiser SM, Tribble CG, Long SM, Kaza AK, Kern JA, Jones DR, et al. Ischemia-reperfusion injury after lung transplantation increases risk of late bronchiolitis obliterans syndrome. Ann Thorac Surg. 2002;73:1041-8.

5. de Perrot M, Liu M, Waddell TK, Keshavjee S. Ischemia-reperfusion-induced lung injury. Am J Respir Crit Care Med. 2003;167:490-511.

6. Wolf PS, Merry HE, Farivar AS, McCourtie AS, Mulligan MS. Stress-activated protein kinase inhibition to ameliorate lung ischemia reperfusion injury. $J$ Thorac Cardiovasc Surg. 2008;135:656-65.

7. Naidu BV, Krishnadasan B, Farivar AS, Woolley SM, Thomas R, Van Rooijen N, et al. Early activation of the alveolar macrophage is critical to the development of lung ischemia-reperfusion injury. J Thorac Cardiovasc Surg. 2003; 126:200-7. 
8. Akira S, Kawai T. TLR signaling. Semin Immunol. 2007;19:24-32.

9. Jiang D, Liang J, Fan J, Yu S, Chen S, Luo Y, et al. Regulation of lung injury and repair by Toll-like receptors and hyaluronan. Nat Med. 2005;11:1173-9.

10. Oppenheim JJ, De Y. Alarmins: chemotactic activators of immune responses. Curr Opin Immunol. 2005;17:359-65.

11. Takeda K, Akira A. TLR signaling pathways. Semin Immunol. 2004;16:3-9.

12. Jeyaseelan S, Manzer R, Young SK, Yamamoto M, Akira S, Mason RJ, et al. Toll-IL-1 receptor domain-containing adaptor protein is critical for early lung immune responses against Escherichia coli lipopolysaccharide and viable Escherichia coli. J Immunol. 2005:175:7484-95.

13. Kim BS, Lim SW, Li C, Kim JS, Sun BK, Ahn KO, et al. Ischemia-reperfusion injury activates innate immunity in rat kidneys. Transplantation. 2005;79: 1370-7.

14. Shimamoto A, Chong AJ, Yada M, Shomura S, Takayama H, Fleisig AJ, et al. Inhibition of Toll-like receptor 4 with eritoran attenuates myocardial ischemia-reperfusion injury. Circulation. 2006;114(1 Suppl):I270-4.

15. Chong AJ, Shimamoto A, Hampton CR, Takayama H, Spring DJ, Rothnie CL, et al. Toll-like receptor 4 mediates ischemia/reperfusion injury of the heart. J Thorac Cardiovasc Surg. 2004;128:170-9.

16. Tsung A, Hoffman RA, Izuishi K, Critchlow ND, Nakao A, Chan MH, et al. Hepatic ischemia/reperfusion injury involves functional TLR4 signaling in nonparenchymal cells. J Immunol. 2005;175:7661-8.

17. Shimamoto A, Pohlman TH, Shomura S, Tarukawa T, Takao M, Shimpo H. Toll-like receptor 4 mediates lung ischemia-reperfusion injury. Ann Thorac Surg. 2006;82:2017-23.

18. Merry HE, Phelan P, Doak MR, Zhao M, Hwang B, Mulligan MS. Role of toll-like receptor 4 in lung ischemia reperfusion injury. Ann Thorac Surg. 2015; doi:10.1016/j.athoracsur.2014.12.062.

19. Shigeoka AA, Holscher TD, King AJ, Hall FW, Kiosses WB, Tobias PS, et al. TLR2 is constitutively expressed within the kidney and participates in ischemic renal injury through both MyD88-depedent and independent pathways. J Immunol. 2007;178:6252-8.
20. Favre J, Musette P, Douin-Echinard V, Laude K, Henry JP, Arnal JF, et al Toll-Like receptors 2-deficient mice are protected against postischemic coronary endothelial dysfunction. Arterioscler Thromb Vasc Biol. 2007;27: 1064-71.

21. Bachar O, Adner M, Uddman R, Cardell LO. Toll-like receptor stimulation induces airway hyper-responsiveness to bradykinin, and effect mediated by JNK and NF-kappa B signaling pathways. Eur J Immunol. 2004;34:1196-207.

22. Droemann D, Goldmann T, Branscheid D, Clark R, Dalhoff K, Zabel P, et al. Toll-like receptor 2 is expressed by alveolar epithelial cells type II and macrophages in the human lung. Histochem Cell Biol. 2003;119:103-8.

23. Krishnadasan B, Naidu BV, Byrne K, Fraga C, Verrier ED, Mulligan MS. The role of proinflammatory cytokines in lung ischemia-reperfusion injury. J Thorac Cardiovasc Surg. 2003;125:261-72.

24. Naidu BV, Krishnadasan B, Byrne K, Farr AL, Rosengart M, Verrier ED, et al Regulation of chemokine expression by cyclosporine A in alveolar macrophages exposed to hypoxia and reoxygenation. Ann Thorac Surg. 2002;74:899-905; discussion 905.

25. Dobbs LG. Isolation and culture of alveolar type II cells. Am J Physiol. 1990 258(4 Pt 1):L134-47.

26. Naidu BV, Farivar AS, Woolley SM, Byrne K, Mulligan MS. Chemokine response of pulmonary artery endothelial cells to hypoxia and reoxygenation. J Surg Res. 2003;114:163-71.

27. Elbashir SM, Harborth J, Weber K, Tuschl T. Analysis of gene function in somatic mammalian cells using small interfering RNAs. Methods. 2002;26: 199-213.

28. McManus MT, Haines BB, Dillon CP, Whitehurst CE, van Parijs L, Chen J, et al. Small interfering RNA-mediated gene silencing in T lymphocytes. J Immunol. 2002;169:5754-60.

Key Words: Ischemia/reperfusion (lung), reoxygenation, TLR-2, TLR-4, proinflammatory mediators

Readers who found these articles interesting may also like to read the following papers found in recent and future issues of our sister publications, Seminars in Thoracic and Cardiovascular Surgery and Operative Techniques in Thoracic and Cardiovascular Surgery!

Evolving Technology/Basic Science

State of the Art: RichardWeisel. The Promise and Challenges of Cardiac Stem Cell Therapy. Semin Thorac Cardiovasc Surg. Spring 2014;26(1):44-52. 